\title{
Performance Characterization of Load Balancing Algorithm
}

\author{
R. Krishan* \\ Department of Computer Science, Mata Sundri University Girls College, Mansa, Punjab, India
}

Received 8 May 2021, accepted in final revised form 27 June 2021

\begin{abstract}
The developing interest in mobile services increases the demand for well-planned and cautiously managed wireless local area networks (WLAN) deployment. In WLAN, a station can access services of the network through an access point (AP) after associating with it. Any number of access points can be accessed by the station whose signal strength is available from among the APs. But practically, a WLAN station (STA) always associates with the access point with higher signal strength among the APs. In WLAN, mobile stations continuously change their location, which results in an uneven network load allocation. This uneven load dissemination prompts an extensive performance degradation of WLAN. This paper presents mathematical modeling to characterize the WLAN performance by balancing the network load and enhancing network throughput. Riverbed Modeler simulator was used to investigate the performance parameters as network load and throughput of the network.
\end{abstract}

Keywords: Wireless local area networks; Mathematical modelling; Performance; Network load; Throughput; Riverbed modeler.

(C) 2021 JSR Publications. ISSN: 2070-0237 (Print); 2070-0245 (Online). All rights reserved. doi: http://dx.doi.org/10.3329/jsr.v13i3.53356 J. Sci. Res. 13 (3), 915-922 (2021)

\section{Introduction}

The performance of the network in WLAN turns out to be significant for various web applications like file, video, data transmission, and concurrent connections, etc. A WLAN structure [1] depicted in Fig. 1 is defined as the set of BSS (Basic Service Set) having access points (APs) with incorporation domain that permits the access of services to its clients by associating with them. The client's requirements with diverse types of applications are distinct in the network. In the network's execution, the distribution of mobile stations among the APs plays an important role in improving the network performance [2]. The highest bandwidth [3] obtainable by an access point is denoted as $\mathrm{C}$.

$$
\mathrm{C}=\mathrm{B} \log _{2}(1+\mathrm{S} / \mathrm{N})
$$

Here $B$ is the rate of data transmission, and $S / N$ is the ratio of signal to noise, and $C$ is calculated in terms of bits/second. Because of obstructions and interferences in a wireless network, the open data transfer capacity to an AP diminishes.

\footnotetext{
*Corresponding author: ramkrishan@pbi.ac.in
} 


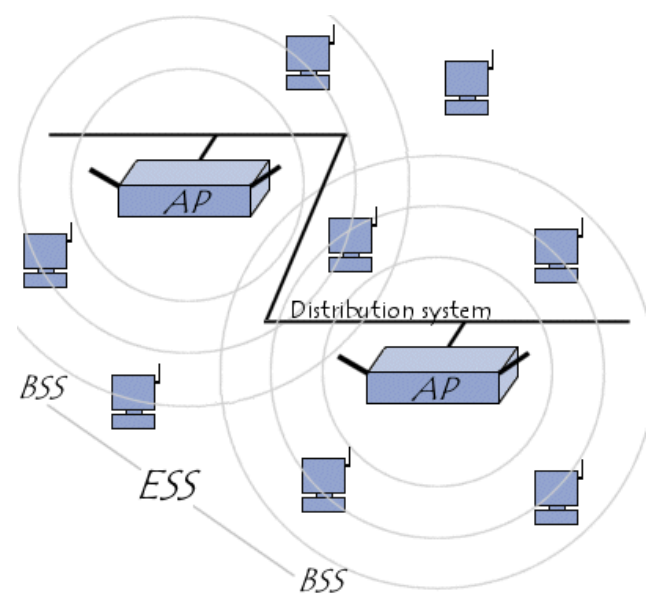

Fig. 1. WLAN structure.

Unfortunately, a WLAN station is always associated with the access point having the highest signal strength without knowing the existing load on an access point (AP). On account of this uneven load distribution [4], some APs remain overloaded, and others stay less-loaded. The goal of our mathematical modeling [5,6] is to balance the network load on APs, by switching the mobile station's load to the other access points available in the range of these stations. With this load balancing, the WLAN throughput is also enhanced.

\section{Related Work}

This section presents the research work done in the area of IEEE 802.11 WLAN load balancing. Gilani et al. developed a mobility management approach, software-defined network, and function virtualization to balance the network load [7]. A mechanism is presented to monitor the load of a real-time network and obtain the improved load balancing performance using a neural network [8]. SDN controller is proposed to analyze the wi-fi load balancing and permit association with the APs. The result shows 34 to $41 \%$ improvement and 28 to $36 \%$ in load balancing degree and re-association time, respectively [9]. Chi et al. developed a new technique of WLAN traffic distribution and AP association. The client association with AP is determined by access points collaboratively. Throughput, transmission delay, and load distribution parameters were analyzed and improved in this approach [10]. Experimental calculation of the performance of the WLAN model and analysis of the different parameters compared to achieve the optimized performance using the OPNET simulator [11]. An optimization algorithm is proposed to distribute the WLAN load. The simulation results of the algorithm were recorded using a riverbed modeler simulator. This solution improves the network performance in terms of network load and throughput [12].

This paper is organized into six sections. Section 1 presents the introduction of the WLAN. Section 2 presents the previous work done in the field of load balancing of the 
WLAN. Section 3 defines the mathematical modeling of the load balancing algorithm. Section 4 shows the network simulation setup and simulation results after implementation, respectively. Section 5 presents the performance characterization of network throughput. Section 6 concludes the paper.

\section{Mathematical Modeling}

The terms and symbols defined for the mathematical modeling of the algorithm are written as: A station (STA) belonging to the network is denoted as $S_{i}$, where $i \in$ $\{1,2,3,---n\}$. The number of access points (APs) in the service area of the network is represented with $\mathrm{AP}_{\mathrm{i}}$, where $i \in\{1,2,3,---n\}$. An access point (AP) range in the network is noted as $R$. $R_{\text {set }}$ is described as the range set of APs in the network. All $S_{\mathrm{i}} \in A P_{i}$ where a station can have a range of one or more access points. A station belonging to the network can be switched to any access points available in $R_{\text {set }}$ to adjust the load of APs in the network as an alternative.

$$
\sum_{i=0}^{n} \mathrm{~S}_{\mathrm{i}} \in \mathrm{AP}_{\mathrm{i}} \quad \forall \mathrm{i} \in\{1,2,3---\mathrm{n}\}
$$

If $\mathrm{S}_{\mathrm{i}} \in \mathrm{AP}_{\mathrm{i}}$

(because of the highest signal strength)

$\mathrm{S}_{2} \in \mathrm{AP}_{2}$

Repeat these steps $\mathrm{n}$ time.

\section{Conditions to execute the algorithm}

$$
\begin{aligned}
& \mathrm{S} \in \sum_{i=0}^{n} \mathrm{AP}_{\mathrm{i}} \quad \forall \mathrm{i} \in\{1,2,3---\mathrm{n}\} \\
& \mathrm{X}_{\mathrm{n}} \in \sum_{i} \mathrm{AP}_{\mathrm{i}} \quad \forall \mathrm{i} \in\{1,2,3--\mathrm{n}\} \\
& \mathrm{S} \text { any AP } \quad \text { (user leave the } \mathrm{n} / \mathrm{w} \text { ) }
\end{aligned}
$$

\section{Execution step}

$\mathrm{S}_{1}, \mathrm{~S}_{2},-----\mathrm{S}_{\mathrm{n}} \quad$ (Comes under $\mathrm{R}$ of $\mathrm{AP}_{1}$ )

$\mathrm{S}_{1}, \mathrm{~S}_{2},----\mathrm{S}_{\mathrm{n}} \rightarrow \mathrm{AP}_{1}$

Check $R_{\text {set }}$ for $S_{\mathrm{i}} \forall \mathrm{i} \in\{1,2,3---\mathrm{n}\}$

Switch $\sum_{i} \mathrm{~S}_{\mathrm{I}} \rightarrow$ other $\mathrm{AP}_{\mathrm{i}} \quad \forall \mathrm{i} \in\{1,2,3--\mathrm{n}\}$ 


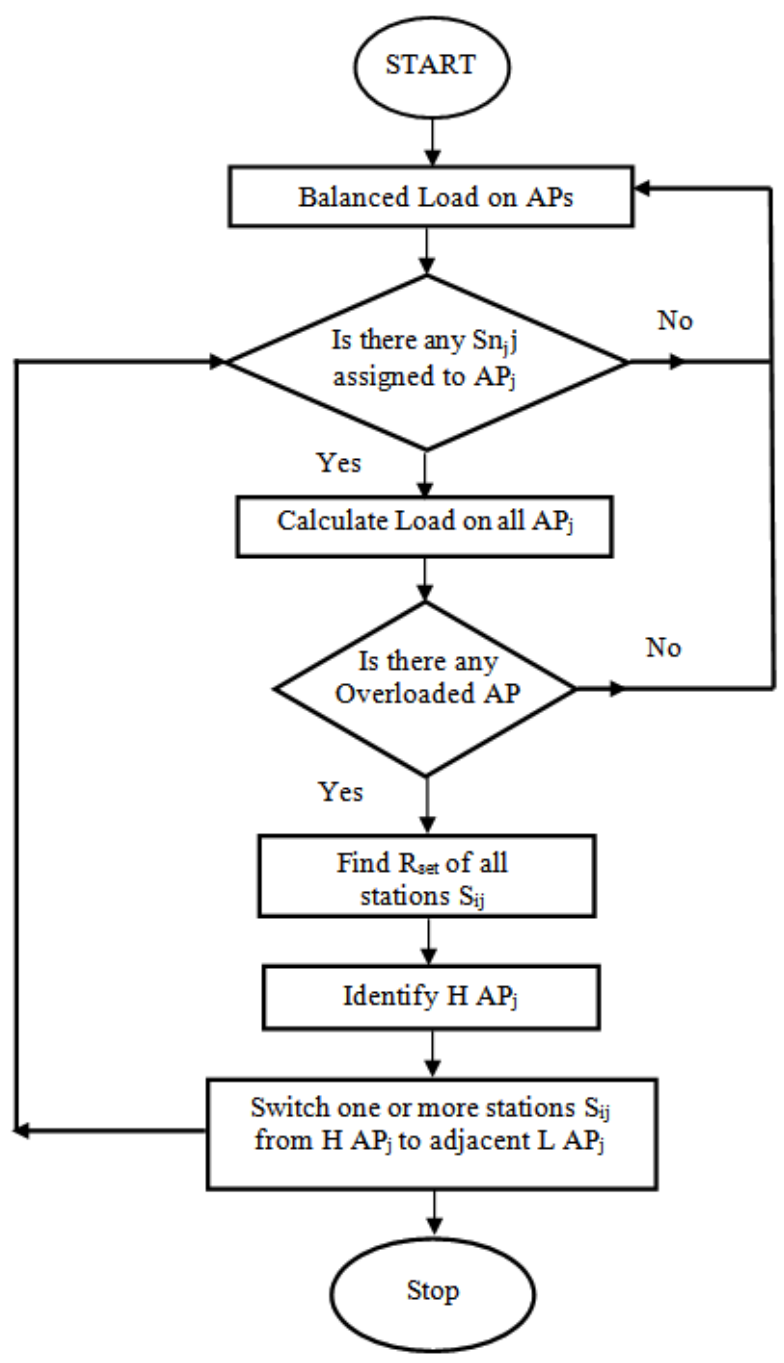

Fig. 2. Flow chart of the load balancing algorithm.

Fig. 2 depicts the computation steps of the load balancing algorithm in the flowchart. At the start, the state of all APs stands balanced. Whenever a new station enters into a network, leaves the network, or associates with another AP due to its mobility, the algorithm initiates its work. The algorithm computes all APs load and checks if any overloaded AP is found; then, the algorithm locates the range set (Rset) of all stations Sij. Further, the algorithm computes the highest loaded AP and switches the stations to the least loaded AP (H APj to adjoining L APj). The algorithm changes the states of the APs after completion of the operation and reiterates this procedure to balance the network load. 


\section{Simulation Setup and Results}

Riverbed Modeler simulator [13] was used to implement and investigate the performance fluctuation of the load balancing algorithm. A WLAN network is designed with 6 BSS and 40 stations (STA). The simulations were performed using the specific application and the profile definition of the network. The results of the simulation were carried out for unbalanced WLAN, balanced WLAN scenario of the network, and recorded for network load and throughput parameters.

\subsection{Network load}

The network load of the WLAN is calculated in bits per second. Fig. 3 depicts the balanced and unbalanced network scenarios with network load on access points of each BSS. Fig. 2 shows the network load of unbalanced and balanced WLAN scenarios graphically. The result of network load on BSS1 in an unbalanced WLAN scenario was $1,62,411$, and in the case of balanced WLAN, it was reduced to 1,48,263 (bits/sec). The result shows a minor increase of load on BSS2 in balanced WLAN from 1,39,502 to $1,51,308$ (bits/sec) compared to unbalanced WLAN. Network load in an unbalanced WLAN scenario on BSS3 was 1,86,316 (bits/sec) and it was recorded as 1,31,177 (bits/sec) in balanced WLAN. Similarly, the network load on BSS4, BSS5, BSS6 in unbalanced WLAN and after running current scenarios in balanced WLAN reduced from $1,40,130$ to $1,20,758$ (bits/sec), $1,76,785$ to $1,51,910$ (bits/sec) and $1,60,158$ to $1,13,118$ (bits/sec) respectively. The graphical result in Fig. 3 shows the balanced distribution of network load and optimizes the network performance.

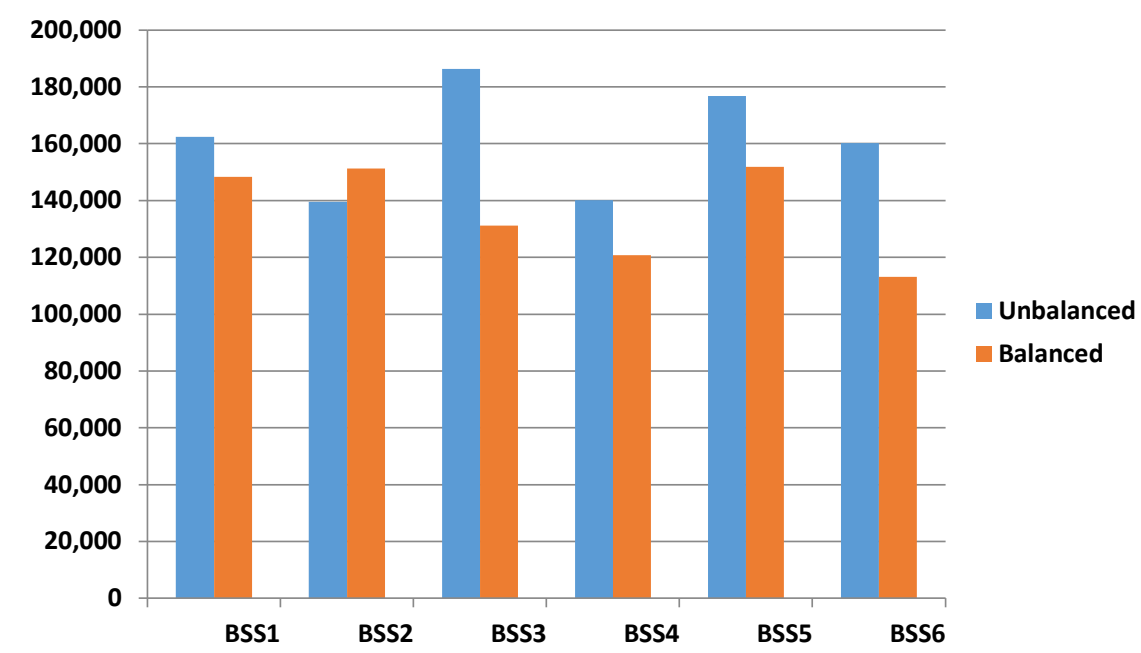

Fig. 3. Graphical representation of network load. 
Table 1. shows the statistical values of network load borne by BSS 1 to BSS 6 in unbalanced and balanced scenarios after simulating, as illustrated in Fig. 3.

Table 1. Statistical network load.

\begin{tabular}{lll}
\hline Basic Service & \multicolumn{2}{c}{ Network load (bits/sec) } \\
\cline { 2 - 3 } Set (BSS) & Unbalanced WLAN & Balanced WLAN \\
\hline BSS 1 & $1,62,411$ & $1,48,263$ \\
BSS 2 & $1,39,502$ & $1,51,308$ \\
BSS 3 & $1,86,316$ & $1,31,177$ \\
BSS 4 & $1,40,130$ & $1,20,758$ \\
BSS 5 & $1,76,785$ & $1,51,910$ \\
BSS 6 & $1,60,158$ & $1,13,118$ \\
\hline
\end{tabular}

\section{Performance Characterization}

For performance characterization of WLAN, the network throughput is compared with Capability-varying AP Technique [10] and Distributed Association Algorithm [14] presented in Figs. 4 and 5. The results show that our algorithm improves the network throughput as compared to the existing algorithm results.

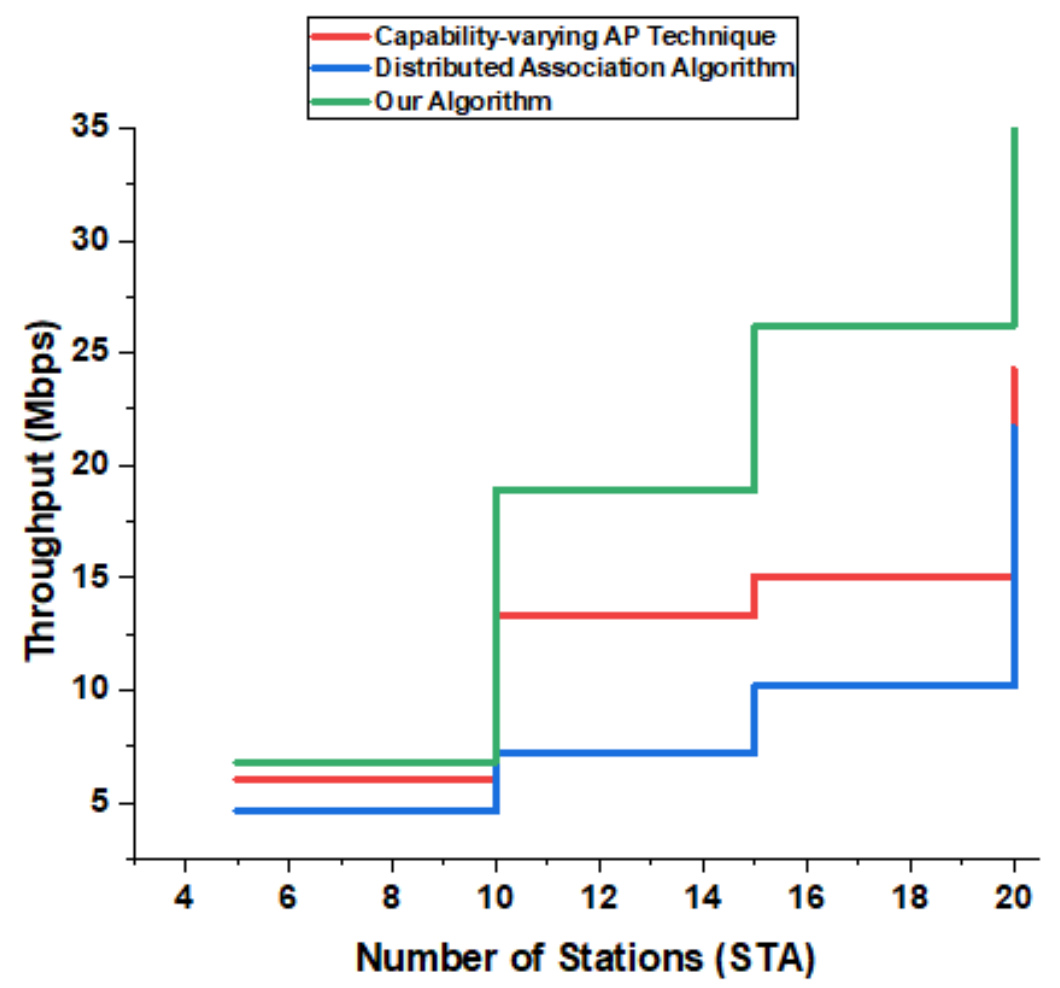

Fig. 4. Case 1 (throughput with maximum 20 stations). 
Table 2. Statistical network throughput with 20 stations.

\begin{tabular}{llll}
\hline $\begin{array}{l}\text { Number of } \\
\text { Stations (STA) }\end{array}$ & $\begin{array}{l}\text { Capability-varying } \\
\text { AP Technique }\end{array}$ & $\begin{array}{l}\text { Distributed Association } \\
\text { Algorithm }\end{array}$ & Our Algorithm \\
\hline 5 & 6.03 & 4.61 & 6.79 \\
10 & 13.34 & 7.16 & 18.92 \\
15 & 15.08 & 10.16 & 26.21 \\
20 & 24.25 & 21.72 & 36.89 \\
\hline
\end{tabular}

Table 3. Statistical network throughput with 40 stations.

\begin{tabular}{|c|c|c|c|c|}
\hline $\begin{array}{ll}\begin{array}{l}\text { Number } \\
\text { stations (STA) }\end{array} & \text { of } \\
\end{array}$ & $\begin{array}{l}\text { Capability-varying } \\
\text { AP technique }\end{array}$ & $\begin{array}{l}\text { Distributed } \\
\text { algorithm }\end{array}$ & association & Our algorithm \\
\hline 10 & 6.15 & 4.48 & & 6.24 \\
\hline 20 & 13.79 & 6.96 & & 22.04 \\
\hline 30 & 14.23 & 10.42 & & 26.89 \\
\hline 40 & 22.23 & 19.77 & & 34.67 \\
\hline
\end{tabular}

Tables 2 and 3 present the statistical result of throughput with 20 and 40 users in different cases. The recorded result shows the overall improvement of network throughput in both cases. The throughput of our algorithm reaches up to 36 and $34 \mathrm{Mbps}$ with a maximum of 20 and 40 stations, respectively.

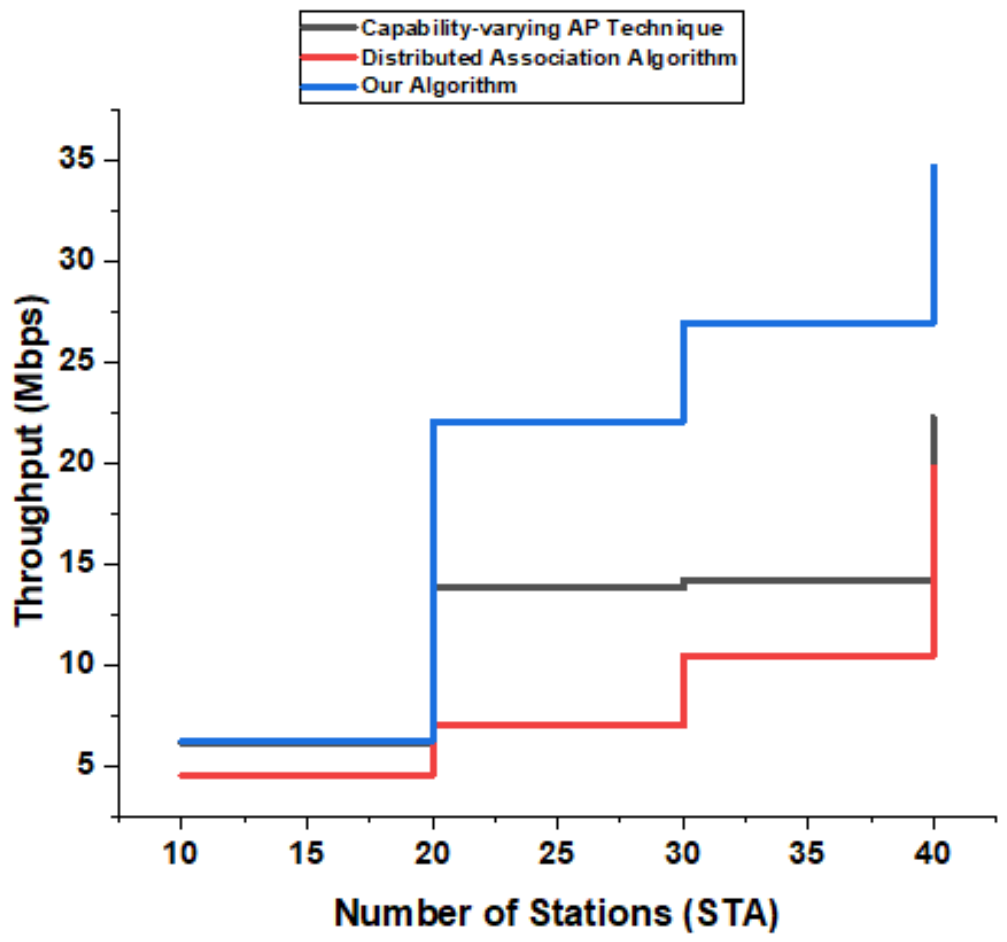

Fig. 5. Case 2 (throughput with maximum 40 Stations). 


\section{Conclusion}

This paper characterizes the performance of WLAN by analyzing the network load and throughput. The simulation results of performance analysis are recorded by using the Riverbed Modeler simulator. By evenly allocating the network load of various stations to the available APs, the algorithm depicts improved performance results. The result shows a balanced network load and improved throughput. The simulation results of network throughput are recorded as 36.89, 34.67 Mbps with 20 and 40 stations in the network. The statistical throughput comparison of our algorithm with the existing algorithm presented in Tables 2 and 3 confirms its suitability for WLAN. In the future, we can extend this work to analyze different parameters related to the WLAN performance and can consider an increasingly intricate environment like hotspots.

\section{References}

1. T. Rappaport, Wireless Communication Principles and Practice (Prentice Hall PTR, USA, 2002).

2. P. Chatzimisios, A. C. Boucouvalas, and V. Vitsas, J. Wireless. Com. Network. 2005, ID 576368 (2005). https://doi.org/10.1155/WCN.2005.67

3. H. Lee, S. Kim, O. Lee, S. Choi, and S. Lee - Proc. Int. Symp. on Modeling, Analysis and Simulation of Wireless And Mobile Systems (ACM, Vancouver, BC, Canada, 2008) pp. 132-139.

4. T. De Schepper, S. Latré, and J. Famaey, J. Netw. Syst. Manage. 28, 133 (2020). https://doi.org/10.1007/s10922-019-09502-2

5. S. Bosio, A. Eisenblätter, H. F. Geerdes, I. Siomina, and D. Yuan, Texts. in Theoretical Computer Science (Springer, Berlin, Heidelberg, 2009) pp. 283-309. https://doi.org/10.1007/978-3-642-02250-0_11

6. T. J. M. Sanguino and J. C. M. Betancourt, Complexity 2018, ID 7560717 (2018). https://doi.org/10.1155/2018/7560717

7. S. M. M. Gilani, T. Hong, and W. Jin, J. Wireless Com. Network. 2017, 67 (2017). https://doi.org/10.1186/s13638-017-0856-9

8. X. Cui, X. Huang, Y. Ma, and Q. Meng, Electronics 8, 273, (2019). https://doi.org/10.3390/electronics8030273

9. Y. D. Lin, C. C. Wang, Y. J. Lu, Y. C. Lai, and H. C. Yang, Wireless. Netw. 24, 2811 (2018). https://doi.org/10.1007/s11276-017-1504-3

10. K. H. Chi and L. H. Yen, Wireless Pers. Commun. 75, 2569 (2014). https://doi.org/10.1007/s11277-013-1488-y

11. T. Wang, H. Zhang, L. Tian, and W. Jiang, Chemical Engine. Trans. 51, 361 (2016).

12. R. Krishan and V. Laxmi, J. Procedia Comp. Sci. 57, 493 (2015). https://doi.org/10.1016/j.procs.2015.07.371.

13. Z. Lu and H. Yang, Unlocking the Power of OPNET Modeler (Cambridge University Press, UK, 2012).

14. H. Gong and J. W. Kim, IEEE Trans. Consum. Electron. 54, 342 (2008). https://doi.org/10.1109/TCE.2008.4560097 